\title{
烷基氟化物消除氟化氢反应的取代基效应 -2
}

\section{李庆明 方德彩 傅孝愿 *}

(北京师范大学化学系, 北京 100875 )

\section{关键词：烷基骕化物，氟化氢，消除反应，取代基效应}

实验发现囟代烷既可以发生 1-1 消除画化氢的反应也可以发生 1-2 消除囟化氢的反 应 ${ }^{[1]}$. 究竟那种消除反应占优势, 主要由取代基团决定。我们曾对氟代烷煌 1-2 热消除反 应的取代基效应进行了研究 ${ }^{[2]}$ 。本文对氪代烷 1-1 热消除氟化氢反应取代基效应进行研 究. 反应式为

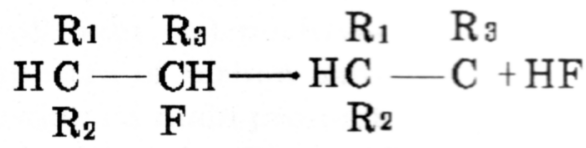

式中 $\mathrm{R}_{1}, \mathrm{R}_{2}, \mathrm{R}_{3}$ 为取代基, 所研究的几个反应物分别为

(1) $\mathrm{R}_{1}=\mathrm{R}_{2}=\mathrm{R}_{3}=\mathrm{H}$;

(4) $\mathrm{R}_{1}=\mathrm{CH}_{3}, \mathrm{R}_{2}=\mathrm{R}_{3}=\mathrm{H}$;

(2) $\mathrm{R}_{1}=\mathrm{F}, \mathrm{R}_{2}=\mathrm{R}_{3}=\mathrm{H}$;

(7) $\mathrm{R}_{1}=\mathrm{CN}, \mathrm{R}_{2}=\mathrm{R}_{3}=\mathrm{H}$;

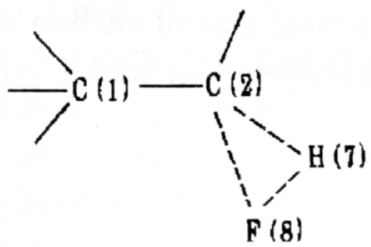

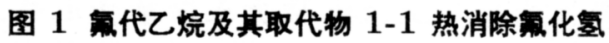
的过渡态的主要原子编号

Fig.1 The numbering system of atoms in the transition states for 1-1 elimination reactions of fluoroethane and substituted fluoroethanes
(5) $\mathrm{R}_{1}=\mathrm{R}_{2}=\mathrm{H}, \mathrm{R}_{3}=\mathrm{CH}_{3}$;

(3) $\mathrm{R}_{1}=\mathrm{R}_{2}=\mathrm{H}, \mathrm{R}_{3}=\mathrm{F}$;

(6) $\mathrm{R}_{1}=\mathrm{R}_{2}=\mathrm{H}, \mathrm{R}_{3}=\mathrm{CN}$;

(9) $\mathrm{R}_{1}=\mathrm{R}_{2}=\mathrm{H}, \mathrm{R}_{3}=\mathrm{NH}_{2}$

(8) $\mathrm{R}_{1}=\mathrm{R}_{3}=\mathrm{H}, \mathrm{R}_{2}=\mathrm{NH}_{2}$;

对反应物的平行态和 1-1 热消除反应的过 渡态, 用 RHF 能量梯度法, 在 3-21G 基 组水平上优化; 用振动分析确认所优化的 过渡态有一个且只有一个负本征值、计算 用 GAUSSIAN 86 程序在 VAX station $4000 / 60$ 工作站上完成.

我们的研究表明 [3]，虽然氟代乙烷 的 1-1 热消除反应产物是开壳层单线态卡 宾，但在其消除反应的过渡态中，基态组 份占 $98 \%$ 。因此这些反应的过渡态是可以 用 RHF 来描述的.

对上述烷基氪化物，1-1 热消除反应 优化的过渡态构型的主要参数见表 1 ，表 1 中的各原子标号如图 1.

1993-09-24 收到初稿，1993-10-16 收到修改稿. 国家自然科学基金资助项目 
奏 1 中的数据表明: 引入吸电子基团 $-\mathrm{CN} 、-\mathrm{F}$ 使得过渡态中 $\mathrm{C}(2)-\mathrm{F}(8)$ 键缩短（见 泰 1 中第 $2,3,6,7$ 列) ; 推电子基团 $-\mathrm{CH}_{3}$ 、 $-\mathrm{NH}_{2}$ 的取代使得过渡态中 $\mathrm{C}(2)-\mathrm{F}(8)$ 键伸长 (事 4,5,8,9 列). 以上四种取代基均使得过渡态中 $\mathrm{H}(7)-\mathrm{F}(8)$ 键伸长. 取代基对消除反应活 保住全的新响见表 2 .

表 1 不同取代基的重代乙烷 1-1 热消除反应过渡态构型的主要参数

Table 1 The chief optimized geometric parameters of the transition states for 1-1 elimination reactions of fluoroethane and substituted fluoroethanes

\begin{tabular}{lccccccccc} 
& \multicolumn{8}{c}{$\mathrm{c}$. } \\
\hline & $\mathrm{R}_{\mathrm{a}}^{*}$ & $\mathrm{R}_{\mathrm{b}}$ & $\mathrm{R}_{\mathrm{c}}$ & $\mathrm{R}_{\mathrm{d}}$ & $\mathrm{R}_{\mathrm{e}}$ & $\mathrm{R}_{\mathrm{f}}$ & $\mathrm{R}_{\mathrm{g}}$ & $\mathrm{R}_{\mathrm{h}}$ & $\mathrm{R}_{\mathrm{i}}$ \\
\hline $\mathrm{C}(1)-\mathrm{C}(2)$ & 1.4940 & 1.4725 & 1.4823 & 1.4931 & 1.4923 & 1.4948 & 1.5024 & 1.4892 & 1.4921 \\
$\mathrm{C}(2)-\mathrm{H}(7)$ & 1.3502 & 1.3517 & 1.2653 & 1.3446 & 1.3225 & 1.3240 & 1.3572 & 1.3406 & 1.1436 \\
$\mathrm{C}(2)-\mathrm{F}(8)$ & 1.9417 & 1.8869 & 1.9170 & 1.9685 & 1.9923 & 1.8881 & 1.8847 & 1.9969 & 2.0804 \\
$\mathrm{~F}(8)-\mathrm{H}(7)$ & 1.0821 & 1.0857 & 1.1464 & 1.0887 & 1.1019 & 1.1035 & 1.0824 & 1.0968 & 1.3306 \\
$\angle 721$ & 119.29 & 117.30 & 121.23 & 118.66 & 116.98 & 118.50 & 118.45 & 115.64 & 115.39 \\
$\angle 821$ & 104.59 & 102.47 & 101.76 & 104.34 & 104.42 & 103.32 & 102.33 & 100.79 & 99.58 \\
$\angle 827$ & 32.50 & 34.40 & 35.25 & 31.83 & 31.27 & 34.90 & 34.37 & 31.16 & 35.68 \\
\hline${ }^{*} \mathrm{R}_{\mathrm{a}}=\mathrm{CH}_{3} \mathrm{CH}_{2} \mathrm{~F}, \quad \mathrm{R}_{\mathrm{b}}=\mathrm{CH}_{2} \mathrm{FCH}_{2} \mathrm{~F}, \quad \mathrm{R}_{\mathrm{c}}=\mathrm{CH}_{3} \mathrm{CHF}_{2}, \quad \mathrm{R}_{\mathrm{d}}=\mathrm{CH}_{3} \mathrm{CH}_{2} \mathrm{CH}_{2} \mathrm{~F}$, & $\mathrm{R}_{\mathrm{e}}=\mathrm{CH}_{3} \mathrm{CHFCH}_{3}$, \\
$\mathrm{R}_{\mathrm{f}}=\mathrm{CH}_{3} \mathrm{CHFCN}, \mathrm{R}_{\mathrm{g}}=\mathrm{CH}_{2} \mathrm{CNCH}_{2} \mathrm{~F}, \mathrm{R}_{\mathrm{h}}=\mathrm{CH}_{2} \mathrm{NH}_{2} \mathrm{CH}_{2} \mathrm{~F}, \mathrm{R}_{\mathrm{i}}=\mathrm{CH}_{3} \mathrm{CHFNH}_{2}$ & &
\end{tabular}

表 2 不同取代基对無代乙烷 1-1 消除反应的活化位垒 $\left(E_{\mathrm{a}}\right)$ 的影响

Table 2 The effect of the substituents on the barrier height $\left(E_{\mathrm{a}}\right)$ for 1-1 elimination reaction of fluoroethanes

\begin{tabular}{cccccccccc}
\hline & $\mathrm{R}_{\mathrm{a}}^{*}$ & $\mathrm{R}_{\mathrm{b}}$ & $\mathrm{R}_{\mathrm{c}}$ & $\mathrm{R}_{\mathrm{d}}$ & $\mathrm{R}_{\mathrm{e}}$ & $\mathrm{R}_{\mathrm{f}}$ & $\mathrm{R}_{\mathrm{g}}$ & $\mathrm{R}_{\mathrm{h}}$ & $\mathrm{R}_{\mathrm{i}}$ \\
\hline$E_{\mathrm{a}} / \mathrm{kJ} \cdot \mathrm{mol}^{-1}$ & 369.5 & 433.5 & 306.9 & 365.6 & 357.3 & 354.1 & 383.2 & 370.3 & 250.3 \\
\hline $\mathrm{R}_{\mathrm{a}}$ etc. as Table 1 & & & & & & & & &
\end{tabular}

由表 2 中的数据可以看出, 虽然在没有取代基的情况下氟代烷的 1-1 热消除相对于 其 1-2 消除是难以实现的 [3]，但在被一定的取代基 (如 $\mathrm{NH}_{2}$ 基) 取代后, 1-1 热消除是可 能的. 这主要是因为 $\mathrm{NH}_{2}$ 的 $\alpha$ 取代使得在 $\mathrm{TS}$ 时, $\mathrm{NH}_{2}$ 中的 $\mathrm{N}$ 与 $\mathrm{C}(2)$ 间距离明显缩短 (从反应物的 $1.4607 \AA$ 到过渡态的 $1.3009 \AA$ ), 因而过渡态较稳定，反应易进行. 表 2 中的数 括表明 $\mathrm{NH}_{2}$ 在 $\alpha$ 位的取代可使 1-1 消除反应的活化位垒降低, 其值与 $\mathrm{NH}_{2}$ 在相同位置取 代的 1-2 消除反应相近 $\left(266.5 \mathrm{~kJ} \cdot \mathrm{mol}^{-1}\right)$, 所以 1-1 和 1-2 消除可以相互竟争. 最近的实验 究 ${ }^{[1]}$ 与我们的这一结论相符.

\section{参考文 献}

1 Rakestraw D J, Holmes B E. J. Chem. Phys., 1991, 95:3968

2 本庆明, 方镘彩, 傅孝愿. 物理化学学报, 待发表.

3 本庆明, 方德彩, 傅孝愿. 科学通报, $1993,38(15): 1440$ 


\section{Theoretical Study on the Substituent Effect of the Elimination of Hydrogen Fluoride from Ethyl Fluoride-2}

Li Qingming Fang Decai Fu Xiaoyuan*

(Chemistry Department,Beijing Normal University,Beijing 100875)

Abstract The substituent effect of the 1-1 elimination reaction of hydrogen fluoride from ethyl fluoride have been studied by $a b$ initio method at the $\mathrm{HF} / 3-21 \mathrm{G}$ level. The reactants are $\mathrm{CH}_{3} \mathrm{CH}_{2} \mathrm{~F}, \mathrm{CH}_{3} \mathrm{CHF}_{2}, \mathrm{CH}_{2} \mathrm{FCH}_{2} \mathrm{~F}, \mathrm{CH}_{3} \mathrm{CH}_{2} \mathrm{CH}_{2} \mathrm{~F}, \mathrm{CH}_{3} \mathrm{CHFCH}_{3}$, $\mathrm{CH}_{3} \mathrm{CHFCN}, \mathrm{CH}_{2} \mathrm{CNCH}_{2} \mathrm{~F}, \mathrm{CH}_{2} \mathrm{NH}_{2} \mathrm{CH}_{2} \mathrm{~F}, \mathrm{CH}_{3} \mathrm{CHFNH}_{2}$ and their reaction barriers are $369.5,433.5,306.9,365.6,357.3,354.1,383.2,370.3,250.3 \mathrm{~kJ} \cdot \mathrm{mol}^{-1}$ respectively. It can be concluded that the 1-1 elimination reaction of $\mathrm{CH}_{3} \mathrm{CHFNH}_{2}$ is the easiest to proceed. This conclusion can be rationalized by its characteristic transition state geometry.

Keywords: Methyl fluoride, Hydrogen fluoride, Elimination reaction, Substituent effect 